\title{
FEATURE \\ Basics of scientific and technical writing: Grant proposals
}

\author{
By Morteza Monavarian
}

This article is the third in a three-part series in MRS Bulletin that will focus on writing papers, patents, and proposals.

\section{Grant proposals}

A grant proposal is a formal document you submit to a funding agency or an investing organization to persuade them to provide the requested support by showing that (1) you have a plan to advance a certain a valuable cause and (2) that the team is fully capable of reaching the proposed goals. The document may contain a description of the ideas and preliminary results relative to the state of the art, goals, as well as research and budget plans. This article provides an overview of some steps toward preparation of grant proposal applications, with a particular focus on proposals for research activities in academia, industry, and research institutes.

\section{Different types of proposals}

There are different types of grant proposals depending on the objectives, activity period, and funding organization source: (1) research proposals, (2) equipment proposals, and (3) industry-related proposals. Research proposals are those that seek funding to support research activities for a certain period of time, while equipment proposals aim for a certain equipment to be purchased. For equipment proposals to be granted, you need to carefully explain how its purchase could help advance research activities in different directions. Unlike research proposals, which are focused on a specific direction within a certain field of research, equipment proposals can have different directions within different areas of research, as long as the proposed equipment can be used in those areas.

There are also industry-related funding opportunities. For example, the National Science Foundation (NSF) has programs within its Division of Industrial Innovation and Partnerships, in which small businesses and industries can involve research funding opportunities. Examples of such programs include Small Business Innovation Research and Small Business Technology Transfer programs. These opportunities are separate from any opportunities directly involving the companies funding your research, where the companies are the source of the funding.

\section{Steps to submit a proposal}

Figure 1 shows an overview of a standard process flow for a grant proposal application, from identifying the needs and focus to acceptance and starting the project. As shown, the process of writing grants is not linear, but rather a loop, indicating the need for consistent modifications and development of your ideas, depending on the input you receive from the funding agencies or the results you obtained from previously funded projects.

Before starting, you need to define the ultimate purpose of the research you want to pursue and to convince others that the work is indeed worth pursuing. Think about your proposed research in the context of problems to solve, potential hypotheses, and research design. To start shaping the idea you are pursuing, ask yourself: (1) What knowledge do I gain from finishing this project? (2) What is the significance of the end goal of the project? (3) How would the completion of this project be useful in a broader sense? Having convincing answers to these questions would be extremely helpful in developing a good grant proposal.

After identifying the needs and focus and initially developing the ideas and plans, the next step is to secure a funding agency to which you would like to submit the grant proposal. It is a good practice to keep track of programs and corresponding funding opportunity announcements for different funding agencies relevant to your field of research. Once you secure a funding agency and find the deadline for submission, review the submission guidelines for the program carefully. The grant proposal document should be perfectly aligned with the structure and content proposed in the guidelines provided by the agency program to avoid any premature rejection of your application. Some programs only require a few documents, while others many more. Some agencies may require a concept paper: a short version of the proposal submitted before you are eligible for a full proposal submission.

After securing the agency/program and reviewing the guidelines, the next step is to write the full proposal document, according to the guidelines proposed by the funding agency. 


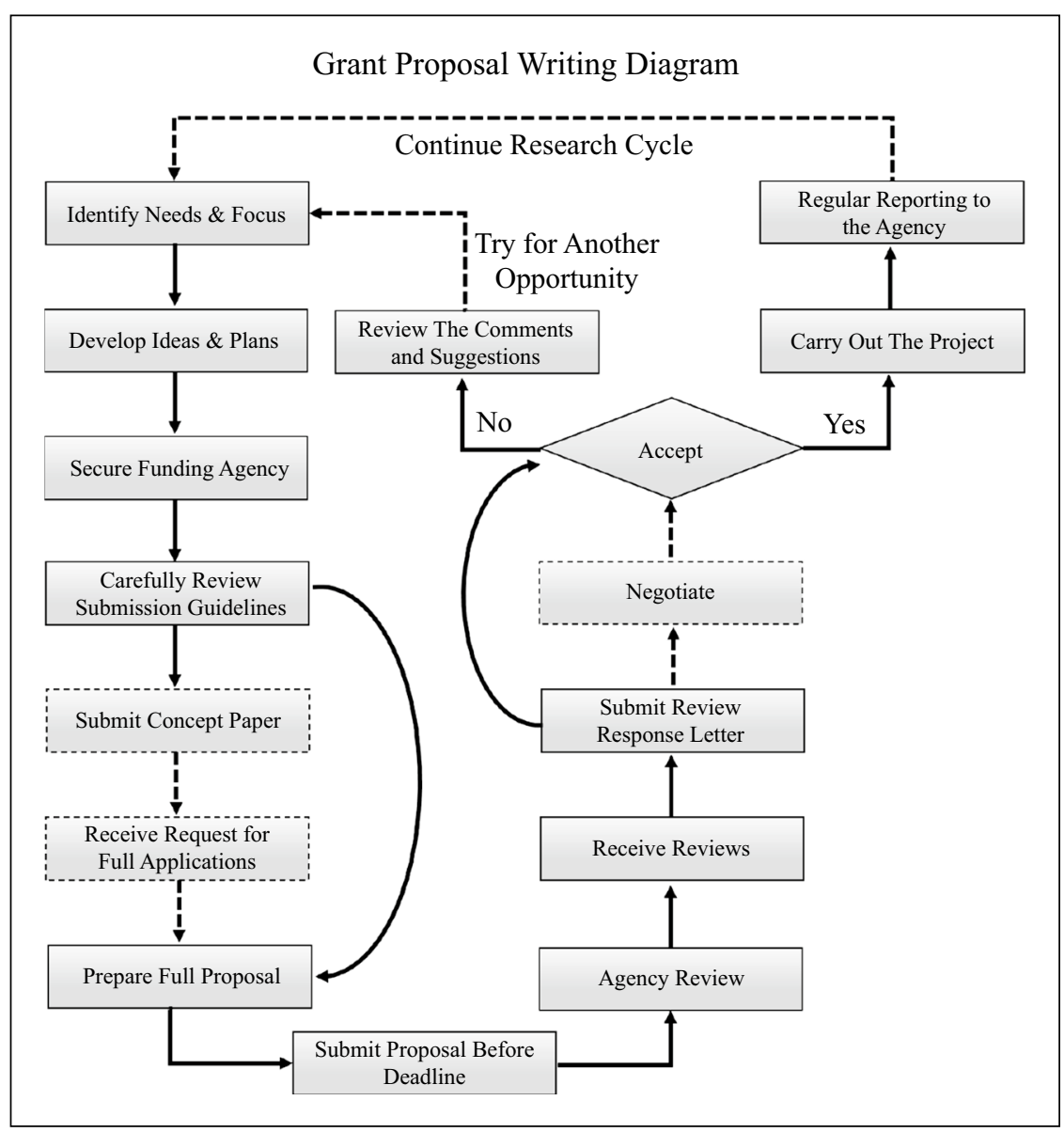

Figure 1. Diagram of grant proposal preparation.

project, agencies normally require a periodic (quarterly or annually) report in either a written or oral form. Different agencies may have rules for any publications or patents that could potentially result during the project term, when the work is complete or the idea is developed as a result of the awarded grant. As shown in the figure, even if the proposal is rejected, upon careful review, revision, and further development or adjustment of the proposal, you may try for another funding opportunity. After finishing a recently funded project, you can further develop an idea and submit another proposal for funding.

\section{Structure of proposals (NSF example)}

The structure of proposals differs with funding agencies. Included is an overview of an NSF proposal as a guide.

In addition to the technical volume (narrative) document, containing all the major descriptions of the project, other necessary documents include bio sketches, budget, justifications, management plan, and project summary. Bio sketches contain resumes of all the principal

Before submission, review your documents multiple times to ensure the sections are well written and are consistent with one another and that they perfectly convey your messages. Some institutes have experts in reviewing proposal documents for potential linguistic and/or technical edits. Submit at least a day before the deadline to ensure that all documents safely go through. Some agencies have strict deadlines, which you do not want to miss, or you may have to wait upwards of a year to submit again. The agency then usually sends your documents to a few expert reviewers for their comments. The review may be graded or have written comments that require attention and response. A response letter has to be prepared and submitted (according to the agency guidelines) by a new deadline imposed by the agency for consideration by the program manager.

After reviewing the full response and revised documents, the agency will contact you with notification of their decision. If your proposal is accepted, the agency will provide details regarding funding and a start date. During the term of the investigators (PIs), including any prior experience, relevant publications, and outreach activities. Budget and justifications are two separate documents relevant to a breakdown of the required budgets for the project, including salaries for the PIs and the team, travel, publication costs, equipment costs, materials and supplies, and any other relevant expenses. The budget document could be an Excel spreadsheet, indicating the exact dollar amounts, while the justification indicates the rationale for each charge. Depending on the agency and program, some expenses are allowed to be included in the budget list (carefully read related guidelines). Other potential requirements for submission may include a description of the project summary, management plans, and the facilities in which the work will be performed.

The technical volume is likely the one you will spend the most time preparing. It consists of several sections. Included is an example of a structure (read the Proposal and Award Policies and Procedures Guide on the NSF website for details). The total technical volume should not exceed 15 pages, excluding 
the reference section, which will be submitted as a separate document. While there are different review criteria for an NSF proposal, the main two are intellectual merit (encompasses the potential to advance knowledge) and broader impacts (potential to benefit society). Your proposal should reflect that the work will be rich in these two criteria. NSF reviewers typically provide qualitative grades (ranging from poor to excellent) to the proposal and feedback in their review.

\section{Introduction and overview}

The first section of the technical volume may start with an introduction/motivation and overview of the proposed work. This section should be no longer than a page, but should give an overview of the background and state of the art in the research area, motivations, objectives of the proposed work (maybe in the context of intellectual merit and broader impacts), and a brief description of the work breakdown (tasks). The last couple of paragraphs of the introduction could summarize the education and outreach plans, as well as the PIs' experience and expertise. Feel free to highlight any major statements in this section to serve as main takeaways for the reviewers. Also, making an overview figure for this section may help summarize the information.

\section{Background and relationship to the state of the art}

The second section gives more details of background and relationship to the state of the art. This section may be a few pages long and contain figures and relevant citations.

\section{Technical methods and preliminary results}

This section should describe the technical methods and preliminary results relevant to the proposed research from your prior work. It should contain illustrative figures and plots to back up the proposed work.

\section{Research plan}

After discussing the prior art and the technical methods and preliminary results (in previous sections), you should discuss the proposed research and plan. A good standard is to divide your work into two to three thrusts, with each thrust containing two to three tasks. You can also prepare a timetable (also called a Gantt chart) to indicate when the tasks will be completed with respect to the project term, which is usually between three to five years.

\section{Integration of education and research}

The last section should describe any plans for integration of education and research, including any K-12 programs or planned outreach activities.

\section{Results from prior supports}

Finally, describe results from all of your prior NSF supports. For each project, provide a paragraph describing the goal of the project, the outcomes, and any related publications. You can also write this section in the context of intellectual merit and broader impacts.

\section{Things to remember when preparing grant proposals}

- Find the proper timing for any idea to explore. Sometimes the idea you think is worth pursuing is either too early or too late to explore, depending on the existing body of literature.

- Begin early to avoid missing any deadlines. Give the process some time, as it could take a while.

- Try to have sufficient preliminary results as seeds for the proposal.

- Have a decent balance between the amount of ideas and preliminary results you put in the grant proposal. Too many ideas but too few results may make your proposal sound too ambitious, while too few ideas and too many results may make your proposed work seem complete, therefore no need for funding.

- Try to attend funding agency panels. It will help you understand the review process, grading criteria, and mindsets of program managers. Learn about proposals that are funded.

- Locate any related funding agency announcements to know the deadlines in advance.

- Be mindful of deadlines. Last day submissions may jeopardize your funding opportunities.

- Learn what is customary. One figure per page is ideal for the proposed technical volume. A wordy proposal with not enough figures will be boring and more difficult for the reviewers to follow.

- Do not give up! You may need to submit several proposals (to different programs/agencies) to get one awarded.

- Be cautious about self-plagiarism! Do not copy and paste texts/figures from your previously supported proposal or papers in your new submissions.

- Be ambitious but practical when developing ideas.

- Develop a solid research program. It is not all about hunting grants; it is also how to execute your funded projects. You may have periods (waves) of grant hunting followed by periods of delivering on the funded projects. Any successful prior research can help you gain more funding in the next wave.

- Enjoy your research!

Morteza Monavarian, Materials Department, and Solid State Lighting \& Energy Electronics Center, University of California, Santa Barbara. 